\title{
La construcción de paz a través de la consolidación del conocimiento biológico y territorial
}

\section{The Construction of Peace through the Consolidation of Biological and Territorial Knowledge}

\author{
Jair Hernando Castro Romero \\ Universidad Federal de São Carlos, Brasil \\ https://orcid.org/0000-0003-0067-5377 \\ Edwin Alexander Sanabria Ospina* \\ Universidad Federal de Integración Latinoamericana, Brasil \\ https://orcid.org/0000-0001-9397-0919
}

Artículo de investigación

Fecha de recepción: 16 de junio de 2019 Fecha de aceptación: 25 de octubre de 2019

\section{Para citar este artículo}

Castro Romero, J. H. y Sanabria Ospina, E. A. (2020). La construcción de paz a través de la consolidación del conocimiento biológico y territorial. Campos en Ciencias Sociales, 8(1), 163-182. Dor: https://doi.org/10.15332/25006681/5719

* Magíster en Ecología y Recursos Naturales de la Universidad Federal de São Carlos, Brasil. Especialista en educación; Ciencia, tecnología y Sociedad, Instituto Federal de São Paulo, Brasil. Correo electrónico: jairhcasrom@gmail.com

** Antropólogo en Diversidad Cultural Latinoamericana de la Universidad Federal de Integración Latinoamericana, Brasil. Correo electrónico: edwinsanabria91@gmail.com 


\section{RESUMEN}

Las sociedades se caracterizan por la construcción de conocimiento colectivo, su manutención y control territorial. La sociedad colombiana está acompañada de una gran biodiversidad, representada en más de 80 tipos de ecosistemas, con cerca del $10 \%$ de la diversidad biológica mundial. Paralelamente, esta también es el modelo económico extractivista y los conflictos socioambientales de nivel local y nacional. Por más de 50 años se desencadenó una guerra armada, que ocasionó pérdidas humanas, culturales y ambientales. Finalmente, el gobierno de Juan Manuel Santos (2010-2018) y las Farc firmaron un acuerdo de paz que toca tangencialmente problemas socioambientales. En este contexto, es necesario promover la sostenibilidad de una ciudadanía que se relacione pacíficamente con el territorio, sus recursos y medios de producción. El presente artículo aborta tres ejes de cuestionamiento: $i$ ) ¿cuáles son los lineamientos educativos en los programas de ciencias biológicas?, ii) ¿qué profesiones integran el debate ambiental en Colombia?, y iii) ¿̨las temáticas tratadas en aula tienen pertinencia con la realidad actual y contribuyen críticamente con el posconflicto? Para resolverlas, se adelantó una búsqueda de los programas reportados en el Sistema Nacional de Instituciones de Educación Superior (sNiEs), de la que se encontraron 250 programas. Extensivamente, cada uno de estos cuenta con amplia formación a nivel disciplinar, pero carece de disciplinas que fomenten la construcción colectiva, interdisciplinar y crítica. En este sentido, el Estado colombiano se encuentra en una etapa diferencial de su historia y los programas académicos no se han transformado para converger en esas nuevas realidades. Debe orientarse la promoción de profesionales que promuevan la paz como una política transversal que se integre a la enseñanza de las ciencias biológicas en la realidad sociopolítica del país y también debe incluirse en la oferta de nuevos programas de posgrado.

Palabras clave: biodiversidad, ciencias biológicas, construcción de paz, educación superior, gestión ambiental.

\section{Abstract}

Societies are characterized by collective knowledge building, its maintenance, and its territorial control. Colombian society is accompanied by great biodiversity, represented in more than 80 ecosystem types, which represent about $10 \%$ of the world's biological diversity. It is also threatened by the extractivist economic model and socio-environmental conflicts at the local and national levels. Colombia had an armed war for more than 50 years, causing human, 
cultural, and environmental losses. Juan Manuel Santos' government (2010-2018) and the Farc signed a peace agreement that touches tangentially on socio-environmental problems. In this context, it is necessary to foster sustainability among the citizens, so that they relate peacefully to its territory, its resources, and production modes. This paper addresses three lines of inquiry: $i$ ) What are the educational guidelines of the biological sciences curricula? ii) What professions partake of the environmental debate in Colombia? iii) Are there topics discussed in the classroom relevant to current reality and contribute critically to the postconflict? To answer this questions, we carried out a search of the curricula reported in the National System of Higher Education Institutions (sNIES) and obtained 250 curricula. These curricula have extensive training at the disciplinary level, but lack the disciplines that foster collective, interdisciplinary, and critical construction. In conclusion, the Colombian State is at a differential stage in its history and academic programs have not been transformed to converge with these new realities. Such programs should be geared toward the promotion of professionals who foster peace as a transversal policy that integrates the teaching of biological sciences into the socio-political reality of the country, and they should also be included in the offer of new postgraduate programs.

Keywords: biodiversity, biological sciences, environmental management, higher education, peace building.

\section{INTRODUCCIÓN}

A lo largo de la evolución cultural, las sociedades han experimentado diversos procesos de cambio y transición en los que se han involucrado diferentes aspectos de la vida humana, su relación con el territorio y los recursos naturales. Paralelamente, la comunidad académica ha explorado relaciones antropológicas de las expresiones culturales y su organización social, así como ha procurado definir y detallar la relación entre las acciones humanas y su contexto natural. El presente trabajo se contextualiza en el proceso de paz de Colombia firmado entre la guerrilla de las Fuerzas Armadas Revolucionarias de Colombia (Farc) y el Gobierno Nacional colombiano, en cabeza del expresidente Juan Manuel Santos Calderón, el día 24 de noviembre de 2016, que trajo para la sociedad colombiana e internacional una nueva lectura del quehacer 
de la paz y la resolución del conflicto armado más violento y antiguo del hemisferio occidental.

El proceso de paz inició durante el año 2012, cuando la guerrilla de las Farc y el gobierno de Juan Manuel Santos comenzaron los diálogos preliminares en Oslo, Noruega. En esta mesa de diálogo se buscaba acercar las posibilidades para acabar el conflicto y comenzar la construcción paz constante y duradera. Según el último reporte de la Unidad de Víctimas (2019), el conflicto armado colombiano tiene alrededor de 8524910 víctimas. De estas, 421942 han sido amenazadas y 1012 201, asesinadas; 11566 fueron víctimas de minas antipersonas; más de 7564146 fueron desplazadas y otras 175153 sufrieron desapariciones forzadas (Red Nacional de Información, 2019), sin contar los actos terroristas, la concentración de tierras y los cultivos ilícitos.

Si bien estos números muestran un conflicto cruel y sanguinario, poco se conoce sobre la relación de la guerra con los recursos naturales. Aunque algunas de las acciones armadas - atentados a redes de petróleo, gas, etc.- produjeron daños ambientales, que afectaron la biodiversidad y el manejo del territorio por parte del Estado y las comunidades locales (Puerta y Dover, 2017). Ha sido evidente la ausencia estructural e institucional del Estado y del mercado, que promueve la manutención de las economías ilegales y organizaciones paralelas.

Dentro de ese contexto, el proceso de paz buscó crear un acuerdo que no solo traería un bienestar bélico, sino para la sociedad colombiana en general (Smitmans y Teresa, 2017). El acuerdo llevaría a la mesa seis puntos fundamentales para lograr la paz con los actores del conflicto armado: i) el fin del conflicto con las Farc; ii) justicia para las víctimas; iii) soluciones al problema de las drogas ilícitas; iv) mejores oportunidades para el campo; $v$ ) apertura democrática, mayor participación, y vi) la implementación, verificación y refrendación del acuerdo.

$\mathrm{Al}$ respecto de los recursos naturales, este constituye un tema transversal a todos los puntos expuestos, ya que, en primer lugar, con la disolución de las Farc como grupo guerrillero dejaría vastas áreas naturales sin el control que ellas mantenían, 
y sin protección ni manejo. Además, al solicitar justicia para las víctimas, las áreas naturales deberían cambiar su actual uso, lo que tiene relación con el tercer punto, puesto que con la disminución de los cultivos ilícitos también caería la deforestación y mejoraría la conservación. En cuarto lugar, desarrollar los derivados del campo generaría una presión sobre los recursos naturales, y por último el aumento de la democracia generaría mayores espacios de deliberación y compromiso comunitario con las áreas naturales y sus servicios ecosistémicos relacionados.

Con el acuerdo de paz, se establecen el fin de los cultivos ilícitos y las nuevas oportunidades para que las poblaciones afectadas por el conflicto obtengan y recuperen tierras, también se propone la recuperación no solamente del territorio, sino de la protección de la biodiversidad. Esta proposición tiene en cuenta diferentes actores sociales como campesinos, investigadores, comunidades étnicas y líderes sociales. Todos ellos entran a jugar un rol fundamental en la protección y cuidado de los diferentes ecosistemas que existen en el país, y sobre todo en zonas de antiguo control guerrillero de las Farc, que impidió por tanto tiempo el desarrollo en investigaciones ambientales, protección de suelos, biodiversidad, etc.

Durante los ciclos de violencia armada, las poblaciones locales se veían sometidas al narcotráfico — cultivo de coca y amapola_-, las cuales eran erradicadas violentamente con fumigaciones de glifosato por el Estado colombiano. Esto acarrea la inhibición de los nutrientes del suelo y lo condena a su deterioro, de manera que la calidad del agua y la salud de las poblaciones que habitaban dichos lugares se vieron afectadas (Bolaños, 2016).

A propósito de esto, la relación entre la naturaleza y la conciencia ambiental debe construirse a través de la exploración y formación del currículo educativo, aunque también es necesario potenciar las prácticas familiares y culturales para que converjan sinérgicamente en ciudadanos con sensibilidad ambiental (Castro, 2018). Con la llegada del posconflicto se abre la posibilidad de repensar las prácticas educativas y sociales, incentivar la investigación de la biodiversidad, así como la conservación del medio ambiente a través de elementos interdisciplinares que compongan la cátedra de paz. Es un espacio de reapropiación territorial y educativa de las comunidades, 
y también de reivindicación de sus prácticas sociales y étnicas. Sin embargo, con la implementación de este nuevo acuerdo de paz y de la elección de Iván Duque a la presidencia de Colombia, se presentan innumerables desafíos políticos en la protección de ecosistemas, flexibilización de normativa ambiental y la violencia contra líderes y lideresas ambientales.

Paulatinamente, la información cotidiana de las regiones quedó secuestrada por la baja difusión, debate e interés urbano. Únicamente fue adoptada por un bajo número de investigadores acoplados a sus especificidades, contrario a las necesidades que el posconflicto plantea, como espacios de construcción colectiva, vinculante y que identifique el territorio con su comunidad.

De esta forma, es evidente que la construcción de la paz se dará a través de la consolidación del conocimiento biológico y territorial. La paz estará arraigada a la memoria - histórica, cultural, social, colectiva-; por lo tanto, es necesario anclarla a la literatura cotidiana, interdisciplinar y de resolución de problemáticas socioambientales. Uno de los objetivos de esta investigación fue analizar y observar el posible manejo de recursos naturales durante el posconflicto, para promover procesos educativos que estabilicen la paz.

Es notable que la salida de grupos armados ha producido inestabilidad en el manejo de recursos naturales y sus derivados, de manera que se han constituido economías ilegales que causan el surgimiento y fortalecimiento de nuevos tipos y formas de violencia. En este sentido, la formación en el manejo de recursos naturales se propone como un eje de formación y manutención de la paz.

\section{Metodología}

Para conocer la dimensión y las características del área de ciencias biológicas en el sistema de educación colombiano se buscaron los programas reportados en el Sistema Nacional de Instituciones de Educación Superior (sNIEs), en cuyo nombre el programa contuviera las palabras: "biología”, "ambiental”, “ambiente”, "ecología”, 
"recursos naturales" y "biotecnología". Posteriormente, los contenidos temáticos de los programas rastreados fueron revisados y clasificados en cuatro categorías: fundamentación científica, disciplinares, optativos y contextos socioambientales. Esta primera aproximación a los programas también tuvo en cuenta la ubicación (región) de las instituciones de educación superior que los ofertan.

La discusión contó con un enfoque cualitativo basado en la necesidad de construcción de paz a través del conocimiento territorial descrito en literatura bibliográfica latinoamericana, específicamente en autores como Leonardo Boff y, a nivel internacional, Michelle Lim. Finalmente se proponen tres caminos estratégicos para contribuir en el posconflicto colombiano.

\section{RESUltados Y DISCUSIÓN}

\section{Ciencias biológicas en el sistema de educación colombiano}

Con el rastreo en la plataforma del SNIES se obtuvieron 250 programas de educación superior, orientados a la enseńanza de ciencias biológicas: 172 de estos programas corresponden al nivel universitario; 60, al nivel tecnológico, y 18, al nivel técnico (figura. 7.1). Este resultado inicial debe cuestionar el tipo de atribuciones y funciones que el estado legisla en el área ambiental y para los cursos de ciencias biológicas.

Da Silva Carneiro y Gastal (2005) ya formulan la complejidad de las ciencias biológicas, desde sus dimensiones, niveles de organización, relación social y construcción moral. En las últimas décadas esta área ha ganado un papel preponderante en la discusión científica hacia la sustentabilidad y contra el antropocentrismo, lejos de presentar un campo homogéneo y coherente ha estado permeado frecuentemente por resistencias, incongruencias y dificultades.

Para los profesionales en ciencias biológicas, en 1986 el Estado colombiano formuló la Ley del Biólogo o Decreto 2531, por medio del cual se reglamenta la Ley 22, sobre el ejercicio de la profesión de la Biología. Después de 34 años de vigencia, existen 
procesos legislativos que deben transformar esta normativa en una ley actualizada que proponga al biólogo como gestor de la biodiversidad, aun considerando sus relaciones antropogénicas, pero manteniendo un enfoque ecocentrista. Lejos del objetivo de esta investigación se encuentran las discusiones epistemológicas sobre el papel de la conciencia ecológica/biológica en la manutención de los servicios ecosistémicos (Rozzi, 1997), que cabe abordar en otros trabajos de investigación.

Figura 7.1. Tipos y cantidad de programas relacionados con las ciencias biológicas en el sistema de educación superior colombiano.

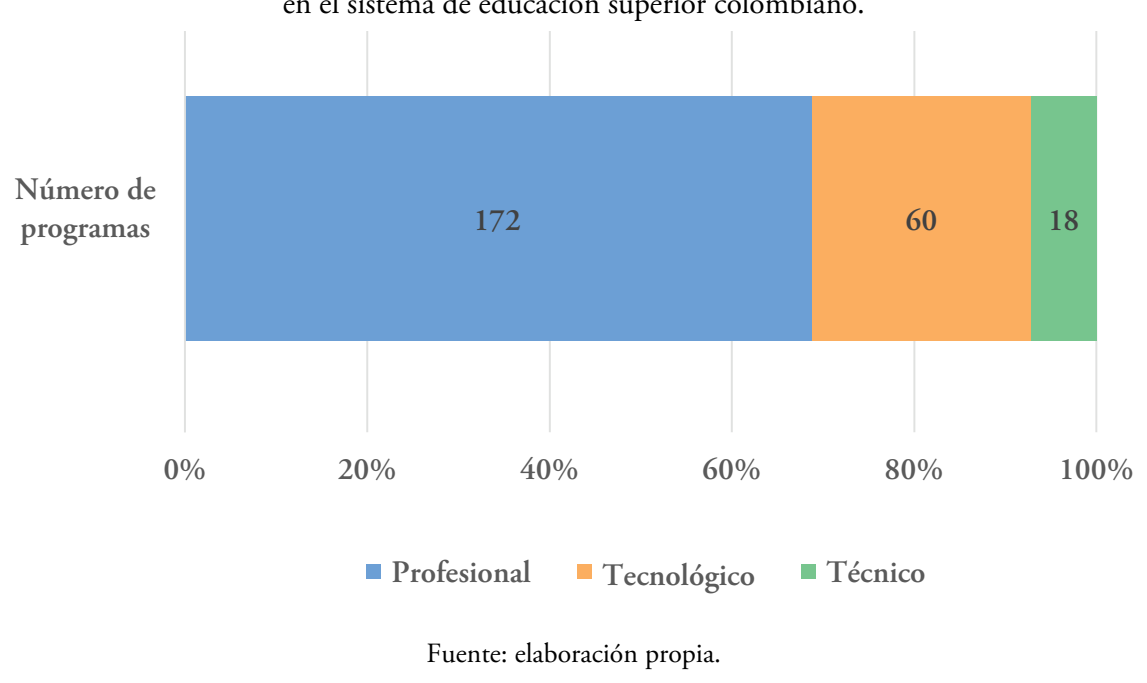

Las atribuciones legales deben partir de discusiones nacionales que se basan en tres ejes:

1. ¿Quién puede decidir sobre los recursos naturales? Este cuestionamiento es de vital importancia, porque los recursos naturales son considerados como bienes particulares. No obstante, la gestión de la biodiversidad promueve la presencia de los actores interesados en la biodiversidad y sus servicios ecosistémicos derivados para mantener altos estándares éticos que promuevan la protección de la salud, turismo, etc. (Corredor, Fonseca y Páez, 2012). 
2. ¿Cuál es el nivel técnico necesario para tomar decisiones? La biodiversidad debe considerarse como un engranaje dependiente de múltiples relaciones ecológicas en diversos niveles de complejidad, que dificultan las visiones radicales y absolutas. Ante esta situación, los tomadores de decisiones deberían presentar una alta cualificación técnica, interdisciplinar, liderazgo y actitud resolutiva (Bodin, 2017).

3. Permanente actualización y en nodos. A lo largo del mundo existen situaciones que pueden resolverse fácilmente utilizando parámetros previos, por ejemplo, la manutención de la paz puede enfocarse desde la resolución efectiva de problemáticas socioambientales (Lim, 2016).

Frente a las áreas de actuación se encontró la siguiente composición: 42, en programas de Biología; 32 son licenciaturas; 13 de Administración; 11 de Microbiología; 4 de Ecología, y finalmente 1 de Biotecnología (figura 7.2).

Figura 7.2. Área de actuación de los profesionales vinculados a programas en ciencias biológicas.

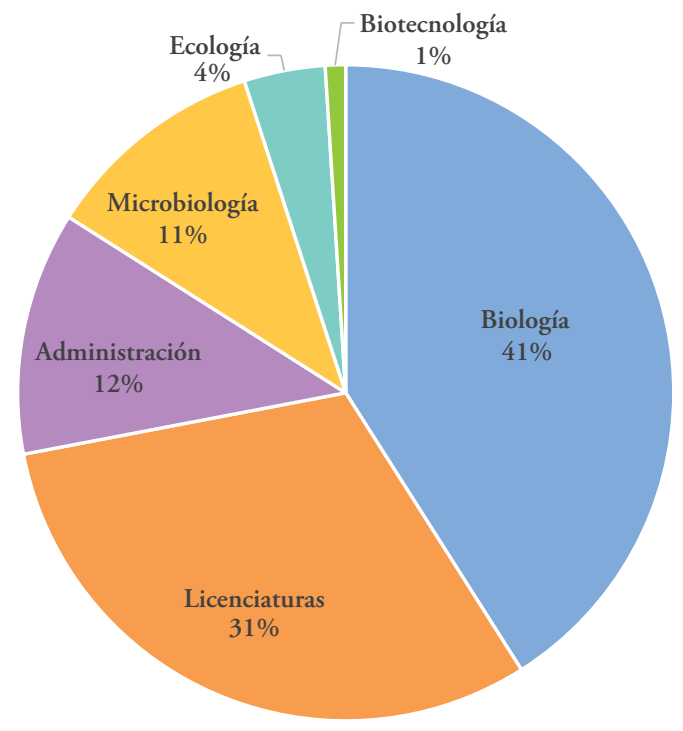

Fuente: elaboración propia. 


\section{Retos vocacionales de los profesionales en el área}

Colombia es considerada el segundo país con mayor biodiversidad del planeta; por ello, se esperaría un gran número de profesionales, así como diversidad en su área de actuación. Más del $70 \%$ de los programas se basan en biología (programas con capacidad de identificación de especies, de investigación ecológica, botánica, zoológica, etc.) y licenciaturas (enseñanza escolar de las ciencias naturales).

Aunque también se considera adecuada la pertinencia de educadores en la escuela, que promuevan didácticas y conciencia ambiental, hay pocos programas administrativos, que tienen en cuenta el manejo de recursos, administración financiera, etc. Finalmente, áreas como microbiología, ecología y biotecnología son residuales, porque son áreas de mayor especificidad, pero de alta innovación y uso mundial. Estas cifras describen un sistema conservador, que difícilmente se transforma, reflexiona y relaciona con las necesidades sociales actuales y en el posconflicto.

\section{Ciencias biológicas en el posconflicto y la coyuntura actual}

Es invaluable la cantidad de información biológica que contienen las áreas liberadas por las Farc (Reardon, 2018): un sinfin de relaciones tróficas, interacciones bióticas y sociales, desconocidas hasta el momento (Clerici et al., 2016). El Instituto de Investigación de Recursos Biológicos Alexander von Humboldt ha motivado la exploración de estas áreas desde diferentes abordajes taxonómicos y científicos, cuyos resultados preliminares muestran un alto número de nuevas especies, con posibles relaciones fitoquímicas y exploración en bioprospección (Páez, 2016).

Paralelamente, se concretó la institucionalización del Ministerio de Ciencia, Tecnología e Innovación (Ley 1951, 2019), el cual se espera que sirva para consolidar la estructura burocrática nacional, la política científica y los principios rectores de la investigación en el país. Para las áreas de posconflicto, servirá en la articulación entre las necesidades sociales y el aprovechamiento de los recursos naturales, y tendrá la posibilidad de potenciar el conocimiento científico, tecnológico o patentes industriales. 
La política científica ingresa en el ciclo de la producción de conocimiento porque se necesita la formación de un alto número de especialistas (magísteres y doctores) que reconozcan nuestra biodiversidad y fomenten investigaciones con un enfoque sostenible y con valor agregado (Morgera y Razzaque, 2017). De lo contrario tendremos la presencia de actores ilegales que generan altos niveles de deforestación, uso insustentable (Teran-Mantovani, 2018) e insostenible de los pobladores locales y la carencia de enfoques de conservación.

Los reportes estatales sobre el posconflicto han evidenciado el aumento de áreas con cultivos ilícitos, lo que recuerda que estas áreas suelen contener un alto grado de biodiversidad, pero son arrasadas por actores ilegales que deforestan ampliamente (Morea, 2017). Esta situación configura otro conflicto, con respecto a la política de drogas y las relaciones de cooperación con Estados Unidos (Nagle, 2002). Esta política promueve el uso de quimio-aspersores (Glifosato) con el objetivo de atacar los cultivos ilícitos, pero estos atacan todos los diferentes niveles de biodiversidad (ecosistemas, especies, genes) y también a las comunidades humanas (Andreotti, et al., 2017).

En esta situación conflictiva, el acuerdo de paz ha promovido la sustitución de cultivos (un proceso que necesita más tiempo, recursos y mayores tasas de manutención) y ha generado choques políticos en la actual coyuntura (Frankel, 2019). Cabe anotar que, si bien la sustitución tiene un enfoque sociopolítico, presenta un trasfondo netamente biológico, ya que las políticas de restauración ecológica y manejo de paisaje son productos específicos de los profesionales en las ciencias biológicas (Handel, 2019).

\section{Desafíos socioambientales}

Por más de sesenta años, la guerra ha dejado un sinfín de desgastes ecológicos en cada una de las áreas donde el conflicto armado hizo presencia. Fácilmente se pueden trazar diferentes conflictos sociales, políticos y ambientales, desde la deforestación masiva, el asesinato de líderes sociales, la minería ilegal y la ausencia estatal (Negret et al., 2019). 
Desde la academia biológica se han adelantado esfuerzos enormes y peligrosos por inventariar lo que se tiene; sin embargo, debido a las circunstancias, estos no han sido lo suficientemente potentes, inclusive con la presencia de especialistas extranjeros.

El andamiaje institucional y político debe promover la creación de nuevo conocimiento, desde la infraestructura, la promoción de posiciones doctorales y posdoctorales, así como espacios de divulgación, inversión en investigación y educación. El fomento de los grupos de investigación y centros interdisciplinares es fundamental para que evalúen las problemáticas ambientales desde diferentes abordajes (Funtowicz y Ravetz, 2018).

Al tener como objeto de estudio a la "biodiversidad", confluyen diferentes relaciones que son congruentes o divergentes en diferentes variaciones; esta dificultad en el abordaje práctico debe tenerse en cuenta al analizar redes tróficas y sus efectos en ecosistemas socioambientales. En este sentido, quien tome decisiones en el ámbito biológico debe tener información de varias áreas de conocimiento, capacidad de negociación e interpelación de intereses.

\section{Construcción de paz}

El concepto de "territorialidad" como una ideología que transforma espacios nacionales, históricos, económicos y de recursos dentro de espacios fronterizos (Paasi, 2011) es transversal a las problemáticas y soluciones regionales. La hipótesis de construcción de paz ambiental es usada frecuentemente en la conservación de recursos naturales, con la cual se argumenta que la cooperación ambiental es un instrumento eficiente para mejorar las relaciones entre diferentes sectores (Conca y Dabelko, 2003). Inclusive, diversos estudios en América Central exploran esta hipótesis, desde diversas perspectivas, en experiencias del Sistema Internacional de Áreas Protegidas para la Paz (Thorsell y Harrison, 1990; Mittermeier et al., 2005; Hammill y Besançon, 2007).

Aunque las políticas de biodiversidad se consideren "suaves", son el punto de partida para negociar y mantener la paz (Downley, 2015). Si bien las áreas protegidas son 
una herramienta de fomento, existen otras dificultades en los sistemas políticos débiles, como baja democracia, centralización y el carácter jerárquico, que acaban socavando la cooperación en el manejo de recursos naturales (Newman et al., 2006).

Un objetivo en este sentido es justamente generar un censo de identidad común zonal, que desencadena un imaginario en el desarrollo de la seguridad comunitaria (Westing, 1998), lo cual conduce a que los conflictos sean más difíciles de imaginar o producirse (Adler, 1997).

Las zonas abandonadas por las Farc contienen los elementos conflictivos que ya se han advertido. En algunas áreas protegidas con riqueza de minerales existen conflictos activos (Barquet, 2015). Otros estudios han argumentado que las áreas protegidas deberían ser un intento para ganar control sobre lugares sin manejo y situados alrededor de las fronteras (Molnar et al., 2001). El caso del Sistema Internacional de Áreas Protegidas para la Paz (SI-A-PAz) refleja el uso de un discurso ambiental para la formación de nuevos territorios.

Al mismo tiempo, la hipótesis de paz ambiental propone construir áreas de conservación como zonas neutrales de cooperación (Barquet, 2015), es decir, como espacios que promuevan los diálogos, acuerdos, y estrategias cooperativas orientadas a la resolución de conflictos.

La resolución de conflictos necesita la presencia de diferentes visiones, abordajes, compromisos, metodologías y acciones. Diariamente es evidente la interrelación entre los conflictos puntuales, como en el caso la minería en áreas naturales y la violencia armada, donde los mecanismos de manutención de las economías ilegales se encuentran como engranajes de violencia. Así como con la minería, existen otros commodities fuera del control estatal y que permean las estructuras financieras delictivas y promueven el financiamiento de la economía ilegal, solventados por el flujo de dinero y medios de consumo, donde existe competencia con la escasa estructura de Estado y las cadenas de mercado inexistentes. 
En Colombia este mecanismo fue utilizado durante los últimos cincuenta años, lo que produjo una sociedad distante de la legalidad, el Estado y la participación como principio democrático. La falta de espacios democráticos conduce al establecimiento de violencias, lo que genera una nueva pregunta: ¿cómo promover la participación democrática en áreas con altos recursos naturales?

La participación social como mecanismo para la conservación biológica es un gran reto, ya que necesita el interés genuino de diferentes actores de la sociedad civil. Para esto deben configurarse leyes que motiven la creación y conformación de consejos participativos, el afianzamiento de sus atributos deliberativos y el mantenimiento de criterios consultivos en creaciones recientes. Esta democratización en la toma de decisiones puede motivar la sostenibilidad, el desarrollo social de las comunidades y la producción de insumos académicos a favor del debate. Por otro lado, el carácter deliberativo deberá ser permitido para que los consejos mantengan una alta participación social, preferiblemente a nivel educativo, jurídico y cultural (Ziccardi, 2004).

La pauta de debate debe construirse desde las múltiples perspectivas para que las decisiones tomadas no perjudiquen algunos sectores. Si bien las empresas privadas deben conformar los espacios de decisión colectiva, es necesario limitar la pauta que únicamente fomente el servicio turístico sin considerar una evaluación de los impactos biológicos o sociales de cada decisión.

Otro punto álgido debe ser el fomento de la investigación en las áreas protegidas y en múltiples temas (Casalet, 2006), como la descripción de biodiversidad en Sudamérica. Su información ancestral, cultural y social debe ser recopilada por científicos locales que promuevan prácticas sustentables y sinteticen la importancia biológica y química de la biodiversidad encontrada.

En las fronteras nacionales esta actividad debe normativizarse para proteger los contactos culturales y los diálogos entre comunidades. La participación debe ser motivada desde la universidad, institutos de investigación, órganos gubernamentales, etc. A su vez, cualquier institución nacional puede promover el interés científico 
como práctica educativa y planear expediciones de carácter exploratorio en las áreas naturales.

Finalmente, la presencia de diferentes actores locales en el sistema de áreas naturales motiva el control social y fomenta la llegada de recursos públicos, nacionales o internacionales, constituidos en otros tratados, que se utilizarían en la constitución de áreas protegidas, lo que llevaría a los siguientes efectos: aumento de personal, construcción de infraestructura para actividades de investigación y turismo sostenible, restauración de áreas impactadas, mitigación de presión ambiental por actividades económicas, optimización de fiscalización, fomento de la educación ambiental en diferentes niveles, y aumento de las extensión de áreas protegidas.

El modelo de representatividad es fuertemente criticado en sociedades altamente diversas como las sudamericanas (Dagnino, 2002). La amplia diversidad cultural, social y política desencadena un alto número de pautas y, en este sentido, una forma de inhibir esta situación es previendo un mayor número de espacios políticos, que permitan aumentar el dinamismo en el control social. La ausencia de comunidades en los espacios de participación social, sea por escaso interés o por falta de infraestructura ciudadana, debe solucionarse por medio del sistema educativo público (Souza, 2001).

La escuela pública debe tener la capacidad de motivar la participación social por medio de la aplicación de los lineamientos curriculares, puesto que aquellos niños que producen y viven procesos de participación podrán aumentar su experiencia y participar activamente en los espacios democráticos de debate durante su juventud. En este sentido, debe ser resaltada la necesidad de un apoyo del sistema educativo que promueva la construcción crítica de conocimiento y mantener la paz.

Otra forma de promoción de la participación social puede darse a partir de la incorporación previa de cada sujeto a las comunidades políticas en formación de lineamientos teóricos, pero este proceso es de menor difusión y volumen que la presencia curricular como eje fundamental de una democracia de derechos. Por esta razón, como política pública para las áreas naturales, se debe aumentar el 
carácter deliberativo y la diversidad de sectores representados en el debate (Thoenig y Durán, 1996).

En este sentido, Brasil motiva la formación de consejos de gestión, en donde participen los diferentes sectores involucrados, lo que da autonomía en la composición del consejo y dinamismo en la estructura orgánica temática, situación que debe ser adaptada a otros países de la región, como en el caso colombiano.

\section{Conclusiones}

La implementación del posconflicto invita a dejar de lado la concepción antropocéntrica y promover un enfoque más ecocentrista, donde la naturaleza tenga un rol fundamental en la conservación de los servicios ecosistémicos no solamente para los humanos, sino para todas las formas de vida. Dicha conservación responde a la cosmovisión de muchos pueblos indígenas amerindios latinoamericanos.

Frente al manejo de recursos naturales, es de vital importancia la formulación e incentivo al desarrollo de mano de obra cualificada, capaz de administrar los recursos naturales de Colombia, el segundo país más biodiverso del mundo; en caso contrario, sin la capacidad institucional, podrían perderse cientos de áreas prácticamente intactas, con especies sin identificar y con potencialidades de investigación e intervención, que pueden ser valiosos para la medicina, la resolución de conflictos, etc.

El Gobierno colombiano, a través del sistema educativo de educación superior y sus universidades, debe repensar los planes curriculares para integrar líneas que incluyan la coyuntura social, las problemáticas, los avances científicos y la paz como hilo tejedor, principalmente considerando el interés que ha surgido en la población durante los últimos años, y que debe ser explorado para la identificación de propuestas que puedan auxiliar la resolución de conflictos. 
Complementariamente, las entidades nacionales deben promover las acciones institucionales, especialmente de carácter biológico, que describan e recopilen inventarios a grandes rasgos de los recursos naturales que están en las áreas abandonadas por las Farc. Con ello, se facilitaría la presencia del Estado, se disminuiría la ilegalidad, se mantendría la paz, se conservaría la biodiversidad y la diversidad cultural de estos territorios, y otros aspectos fundamentales para el respeto a los derechos humanos y de la tierra.

Finalmente, la paz es un concepto abstracto que se materializa a través de la comunicación, el manejo y la negociación, los recursos naturales como fuente financiera de las economías ilegales suelen financiar los conflictos y su violencia relacionada. Ante esto es necesario promover la literatura como medio para la reconstrucción histórica y la memoria cultural, con lo cual se consolida el conocimiento biológico y territorial, como parte de los esfuerzos a la construcción cotidiana de paz.

\section{Agradecimientos}

Esta investigación se ha producido gracias al interés genuino de Jair Hernando Castro Romero y a Kristhy A. Cobo Soto, que ha sido parte de las temáticas incluidas en la disciplina sobre tópicos biológicos en el contexto latinoamericano. Los autores agradecen a Samantha Esparza Naranjo y Anthony Muñiz Bravo por su contribución en la recolección bibliográfica y también a Katherine Patiño por su revisión en el desarrollo de este trabajo.

\section{REFERENCIAS}

Adler, E. (1997). Seizing the middle ground: Constructivism in world politics. European Journal of International Relations, 3(3), 319-363. 
Andreotti, G., Koutros, S., Hofmann, J. N., Sandler, D. P., Lubin, J. H., Lynch, C. F., Lerro, C. C. ... Freeman L. E. (2018). Glyphosate Use and Cancer Incidence in the Agricultural Health Study. JNCI: Journal of the National Cancer Institute, 110(5), 509-516.

Barquet, K. (2015). "Yes to Peace"? Environmental peacemaking and transboundary conservation in Central America. Geoforum, 63, 14-24.

Bodin, O. (2017). Collaborative environmental governance: Achieving collective action in social-ecological systems. Science, 357(6352), 63-52.

Bolaños Muñoz, E. (2016). La "suspensión" del glifosato en la erradicación de cultivos ilícitos en Colombia: ¿una solución humanitaria o un cambio para que todo siga igual? [trabajo de grado]. Universidad Católica de Colombia. Bogotá, Colombia.

Casalet, M. (2006). Las nuevas tendencias en la organización y financiamiento de la investigación. Seminario Internacional Globalización Conocimiento y Desarrollo, unAM, México.

Castro Romero, J. H. (2018). Interfaces entre la conservación de la naturaleza y las políticas educativas latinoamericanas [trabajo de grado]. Universidade Federal da Integração LatinoAmericana. Foz do Iguaçu, Brasil.

Clerici, N., Richardson, J. E., Escobedo, F. J., Posada, J. M., Linares, M., Sánchez, A. y Vargas, J. F. (2016). Colombia: Dealing in conservation. Science, 354(6309), 190.

Congreso de la República de Colombia. (1986, 4 de agosto). Ley del Biólogo [Decreto 2531 de 1986]. Diario Oficial n. ${ }^{\circ} 31490$.

Congreso de la República de Colombia. (2019, 24 de enero). Ley 1951 de 2019. Diario Oficial n. ${ }^{\circ} 50853$.

Corredor E. S., Fonseca J. A. y Páez E. M. (2012). Los servicios ecosistémicos de regulación: tendencias e impacto en el bienestar humano. Revista de Investigación Agraria y Ambiental, 3(1), 77-83. 
Da Silva Carneiro, M. H. y Gastal, M. L. (2005). História e filosofia das ciências no ensino de biologia. Ciência \& Educação, 11(1), 33-39.

Dagnino, E. (2002). Sociedade civil, espaços públicos e a construção democrática no Brasil: limites e possibilidades. En Sociedade civil e espaços públicos no Brasil (pp. 279-301). São Paulo: Paz e Terra.

Frankel, M. (2019). Avocado Root Rot: Steep, Rocky Terrain and Biodiversity Help Protect Small Farmers in Post-Conflict Colombia [tesis de maestría]. San Jose State University, California, Estados Unidos.

Funtowicz, S. y Ravetz, J. K. (2018). Post-normal science. En N. Castree, M. Hulme y J. Proctor (eds.), The Companion to Environmental Studies (pp. 443-447). London: Routledge; GSE Research.

Handel, S. N. (2019). The Living Dead and the Practice of Landscape Restoration. Ecological Restoration, 37(3), 140-141.

Lim, M. (2016). Governance criteria for effective transboundary biodiversity conservation. International Environmental Agreements: Politics, Law and Economics, 16(6), 797-813.

Morea, J. P. (2017). Territorial Conflicts and Conservation of Biodiversity in Protected Areas of Argentina. Investigaciones Geográficas, (68), 115-132.

Morgera, E. y Razzaque, J. (eds.). (2017). Biodiversity and Nature Protection Law (vol. 3). Cheltenham: Elgar Publishing.

Negret, P. J., Sonter, L., Watson, J. E., Possingham, H. P., Jones, K. R., Suarez, C., ... Maron, M. (2019). Emerging evidence that armed conflict and coca cultivation influence deforestation patterns. Biological Conservation, 239, 108-176.

Páez, V. P. (2016). Colombia's tax on wildlife studies. Science, 354(6309), 191. 
Puerta Silva, C. P. y Dover, R. V. (2017). Salud, recursos naturales y el proceso de paz en colombia. The Journal of Latin American and Caribbean Anthropology, 22(1), 183-188.

Reardon, S. (2018). Farc and the forest: Peace is destroying Colombia's jungle--and opening it to science. Nature, 558(7709), 169-170.

Red Nacional de Información. (2019). Reporte general de víctimas. Consultado en https:// cifras.unidadvictimas.gov.co/Home/General.

Rozzi, R. (1997). Hacia una superación de la dicotomía biocentrismo-antropocentrismo. Ambiente y Desarrollo, 13(3), 80-89.

Smitmans, A. y Teresa, M. (2017). El Proceso de Paz en Colombia: dos pasos adelante, un paso atrás. Estudios Internacionales, 49(187), 163-179.

Souza, C. (2001). A nova gestão pública. En Gestión pública: desafíos y perspectivas (pp. 3862). Salvador: Fundación Luís Eduardo Magalhães.

Teran-Mantovani, E. (2018). Inside and beyond the Petro-State frontiers: geography of environmental conflicts in Venezuela's Bolivarian Revolution. Sustainability Science, 13(3), 677-691.

Thoenig, J. C. y Durán, P. (1996). L'Etat et la gestion publique territoriale. Revue française de science politique, 46(4), 580-623.

Westing, A. H. (1998). Establishment and management of transfrontier reserves for conflict prevention and confidence building. Environmental Conservation, 25(2), 91-94.

Ziccardi, A. (2004). Espacios e instrumentos de participación ciudadana para las políticas sociales del ámbito local. En Participación ciudadana y políticas sociales en el ámbito local (pp. 245-272). México, D. F.: Universidad Autónoma de México. 